\title{
Optimization of Channel for Helium Cooled DEMO First Wall by Application of One-sided V-shape Ribs
}

The plasma facing first wall (FW) of a fusion power reactor has to absorb high heat fluxes from the plasma. Helium gas as a coolant offers several advantages in terms of safety. However the use of standard smooth cooling channel surfaces are limited regarding the cooling of high heat flux components in fusion power reactors. Based on our previous assessments, a roundedged, one-side-ribbed rectangular channel was chosen as the baseline geometry, with the ribbed-side facing the plasma-facing wall. Optimizations were done on a V-shaped one-side-ribbed channel by means of CFD simulation, focusing on the rib pitch, rib angle and channel height.
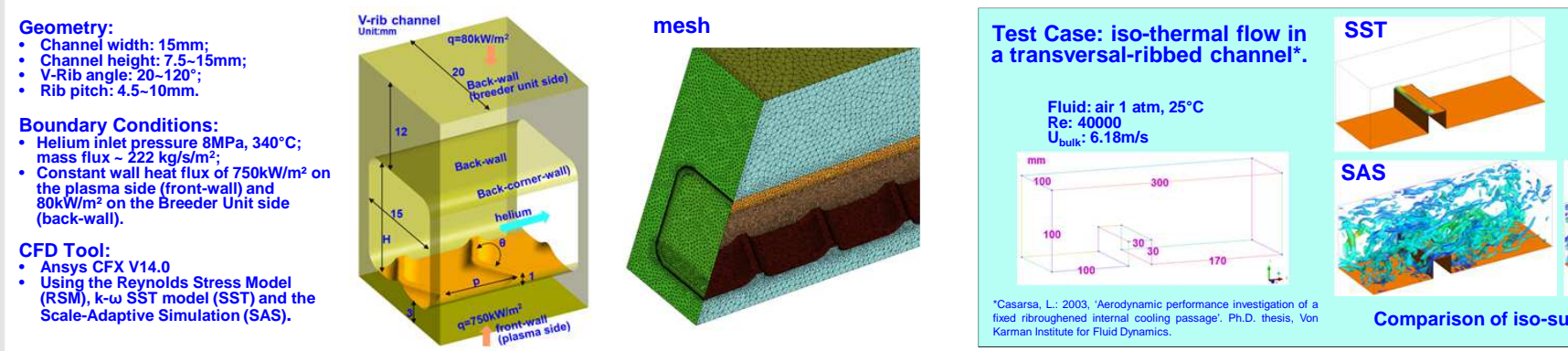

RSM
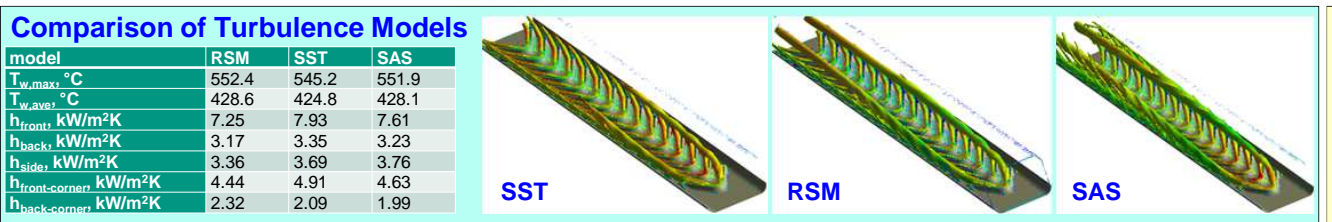

Flow \& Temperature Fields
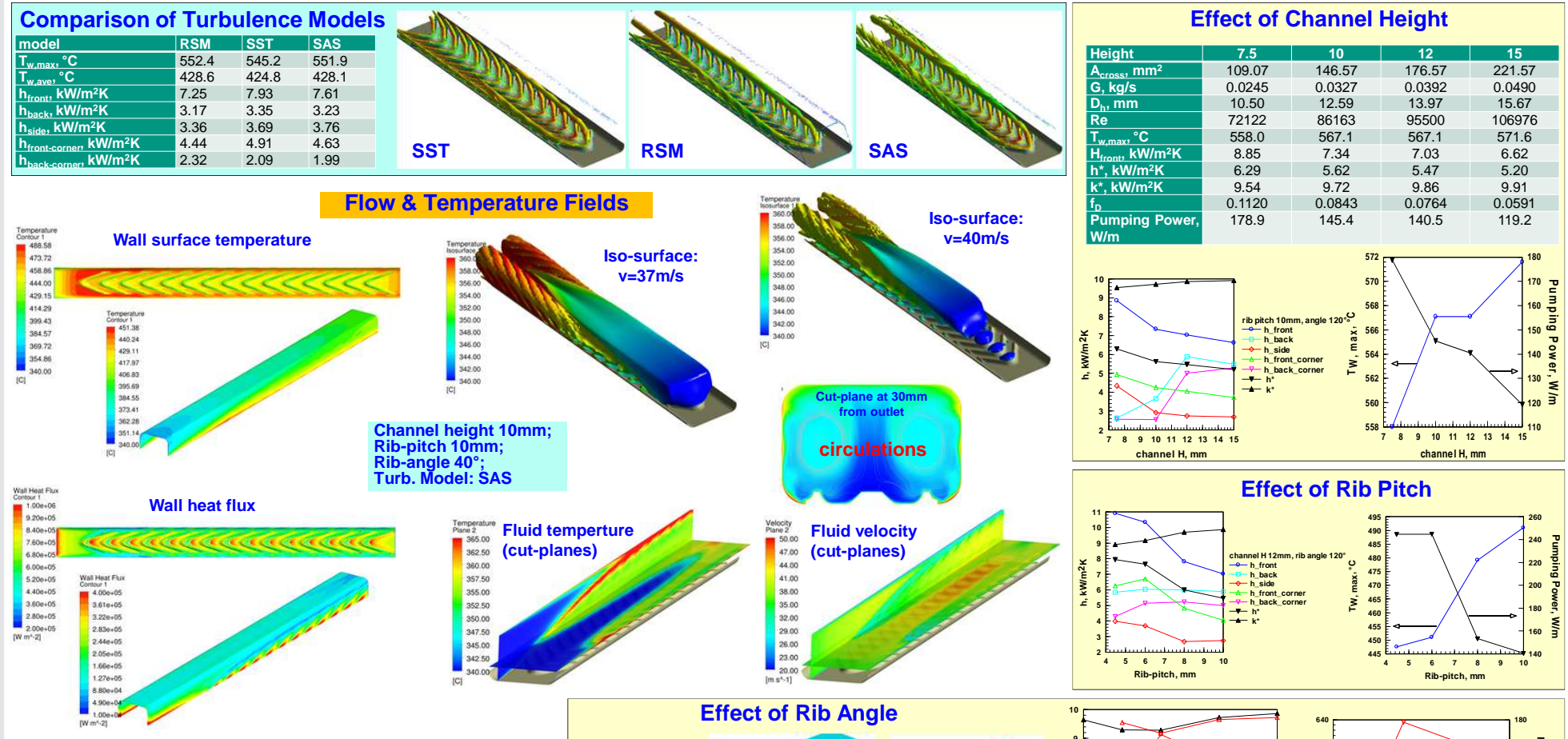

\section{Comparison of smooth, transversal ribbed and V-ribbed channels}

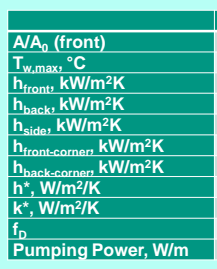

\begin{tabular}{|c|c|c|}
\hline smooth & Transversal-rib & V-rib \\
\hline 1.00 & 1.22 & 1.13 \\
659.1 & 578.9 & 567.1 \\
\hline .87 & 5.48 & 7.03 \\
4.77 & 6.52 & 5.88 \\
3.09 & 3.21 & 2.74 \\
2.63 & 4.18 & 4.05 \\
4.42 & 5.67 & 5.00 \\
3.14 & 5.01 & 5.47 \\
11.28 & 1.01 & 9.86 \\
0.0276 & 0.0708 & 0.0764 \\
\hline 50.6 & 130.3 & 140.5 \\
\hline
\end{tabular}
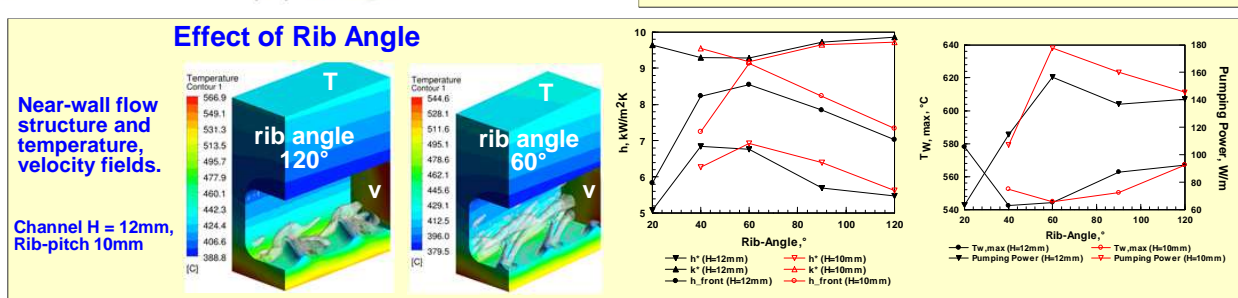

$>$ The effect of the channel height on the maximum wall temperature is relatively small; the decrease of rib pitch leads to decreasing of maximum wall temperature, while also leads to a relatively large increase of pumping power.

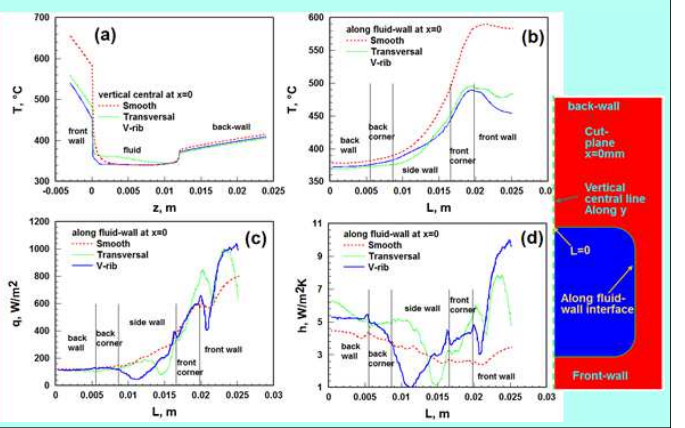

$>$ The modification of rib angle can be made to reduce the wall temperature without significantly increasing the pumping power.

$>$ Overall, the V-ribbed channel with a channel height of $10 \mathrm{~mm}$, rib pitch of $10 \mathrm{~mm}$ and rib angle of $40^{\circ}$ is one of the favorable configurations, which can achieve a heat transfer enhancement by a factor of 2.8 compared to the smooth channel and reduces the maximum wall temperature by about $100^{\circ} \mathrm{C}$.

A major mechanism of heat transfer enhancement for the V-ribbed channels (with the "V" pointing upstreams) is the two large scale circulations induced by the two legs of the Vribs. Therefore, it is believed that for a given coolant flow rate there is an optimal rib angle.

$>$ The SAS model can be used for predicting the flow in ribbed channels. The RSM and SST models are unable to reveal the turbulent structures; while the RSM model can provide similar predictions of temperature and heat transfer coefficient as the SAS model. 